\title{
Addendum
}

\section{Gold-Catalysis: Reactions of Furandialkynes}

A. Stephen K. Hashmi, * Elisabeth Enns, Tanja M. Frost, Sascha Schäfer, Wolfgang Frey, Frank Rominger Synthesis 2008, 2707.

The corresponding author Andreas M. Schuster was missing from the list of authors. The full author list should be:

A. Stephen K. Hashmi, * Elisabeth Enns, Tanja M. Frost, Sascha Schäfer, Andreas M. Schuster, Wolfgang Frey, Frank Rominger 